\title{
Hypotensive central infarction of the spinal cord
}

\author{
P C BLUMBERGS AND E BYRNE
}

From the Departments of Neurology and Neuropathology, The Royal Adelaide Hospital and Institute of Medical and Veterinary Science, Adelaide, South Australia

SUMMARY A case of selective necrosis of the central grey matter of the caudal spinal cord secondary to severe prolonged hypotension is presented. The hypotension was due to localised dissection of the ascending aorta which did not interfere structurally with any of the spinal cord vasculature.

Spinal cord injury in dissecting aneurysms of the aorta usually results from occlusion or severance of intercostal and lumbar arteries which are of vital importace to the blood supply of the spinal cord. ${ }^{1}$ The spectrum of resultant cord damage varies from ischaemic changes limited to a few ganglion cells to complete necrosis of grey and white matter. ${ }^{2}$ In some cases, an unusual selective central grey matter infarction $^{2-5}$ has been described, the pathogenesis of which is not well understood.

\section{CASE REPORT}

A 68-year-old caucasian male underwent quadruple coronary artery saphenous vein bypass grafts for relief of persistent angina following an acute inferior myocardial infarction. The post-operative course was uneventful, except for an episode of exertional angina three weeks after the operation followed one week later by the sudden development of severe dyspnoea and circulatory collapse. For ten hours he remained severely hypotensive with systolic blood pressures ranging from 70 to 90 $\mathrm{mmHg}$. At this stage a dense flaccid paraparesis was noted with sensory levels to pin prick at T12 on the right and T7 on the left. Sixteen days after the cnset of paraparesis, the patient developed severe central chest pain and died a few hours later.

At autopsy a localised dissection of the ascending aorta was found which had extended into the right atrial wall to produce a large intramural haematoma compressing the proximal superior vena cava. An intimal tear was present in the posterior wall

Address for reprint requests: Dr PC Blumbergs, Institute of Medical and Veterinary Science, Box 14, Rundle Street Post Office, Adelaide, South Australia, 5300.

Acsepted 11 April 1980 of the aorta commencing at the posterior margin of the uppermost graft and descending to the level of the aortic valve cusps. Histological examination at the margins of the haematoma showed organising granulation tissue consistent with rupture 16 days earlier. All the grafts were patent. The coronary arteries showed severe atheroma and the right coronary artery was thrombosed proximal to the skip graft. A $10 \mathrm{~mm}$ recently healed infarct was present in the posterior wall of the left ventricle. The aorta was severely involved with ulcerative atheroma. The intercostal arteries were normal. The ostia of the lumbar and iliolumbar segmental arteries were severely stenosed by atheroma, but none were completely occluded.

Neuropathology Careful examination of the formalinfixed brain revealed no abnormality and in particular there was no evidence of damage in the "watershed" areas or in the areas especially vulnerable to hypoxia.

The vertebral column was removed with the spinal cord in situ and fixed in $10 \%$ formol-saline. The blcod vessels were carefully examined both in situ within the vertebral canal and after removal of the spinal cord. The medullary arteries including the great anterior medullary artery of Adamkiewicz (left eleventh thoracic anterior nerve roots) and the anterior median and posterior spinal arteries were patent. Tissue blocks were obtained from each segmental level, embedded in paraffin and stained with haematoxylin-eosin, cresyl violet, Weil and Glees and Marslands method for axis cylinders. Examination showed central grey matter necrosis extending from T8 to S5 (fig 1a) with maximal involvement at L4-S5 levels where there was symmetrical total loss of neurones and neuroglia and replacement by foamy macrophages (fig 1b). In segments $T 11$ to L3 there was partial sparing of grey commissures and in T8-T10 the necrosis was limited to the posterior horns. The white matter 


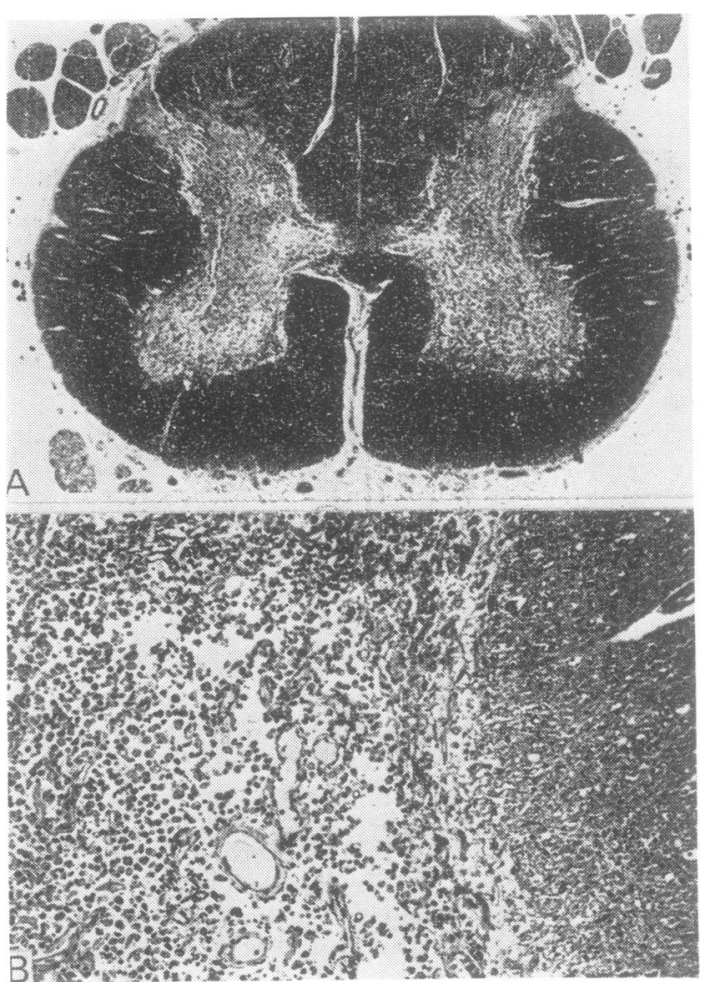

Fig 1 (A) L3 spinal cord showing ischaemic necrosis of the central grey matter. (Weil stain for myelin.) (B) Junction of grey and white matter (L3) showing replacement of grey matter by numerous foamy macrophages and intact white matter. (Haematoxylin-eosin $\times 56$.)

was normal, except for a few swollen eosinophilic axis cylinders and rare foamy macrophages immediately adjacent to the areas of maximal necrosis. The intrinsic spinal cord vessels were all patent.

\section{Discussion}

Selective necrosis of the central grey matter of the spinal cord has been reported in two groups of cases: those associated with disruption or occlusion of segmental vessels and those following cardiac arrest. In the first group, ${ }^{2-5}$ the selective grey matter necrosis involved the lower thoracic and lumbosacral segments of the spinal cord and was due to severance of intercostal and lumbar arteries, ${ }^{23}$ or surgical clamping of the thoracic aorta. ${ }^{4}$ Herrick and Mills ${ }^{5}$ reported two cases of selective grey matter necrosis secondary to asymptomatic aortic disease. Their first patient had suffered a painless dissecting aortic aneurysm which had occluded the left 9th, 10th and 11th intercostal arteries and in their second case many of the small vessels were occluded by cholesterol emboli which were believed to have arisen from a severely atheromatous aorta.

In the second group, Gilles and $\mathrm{Nag}^{6}$ examined the spinal cords of six children who had suffered sudden hypotension following cardiac arrest and observed subtotal symmetrical necrosis of the anterior grey matter which was most severe in the caudal spinal cord. Schneider, Dralle and Ebhardt ${ }^{7}$ described subtotal necrosis of the grey matter in four adults subsequent to circulatory arrest. This apparent vulnerability of the lumbosacral spinal cord was also observed by Azzarelli and Roessmann ${ }^{8}$ who studied the spinal cords of sixteen patients who had suffered from "anoxic" episodes but in none of their cases was there total selective necrosis of the central grey matter.

While the pattern of the lesions produced in the present case is similar to that described above, the mechanism of perfusion failure appears to be different. There had been no structural interference with the segmental vessels and there was no evidence of cardiac arrest or hypoxic brain damage. Paraplegia has been described in relation to occlusion of the coronary arteries, ${ }^{9}$ but the pathological picture was different in that both grey and white matter infarction occurred on a presumed embolic basis. It is uncertain whether a relationship exists in our case between the surgical procedure and the subsequent development of aortic dissection.

We believe that, in this case, the caudal spinal cord was critically dependent on the longitudinal vascular plexus for its blood supply because of the ostial narrowing of the lumbar and iliolumbar segmental vessels. When hypotension occurred the lumbosacral cord, being most distal, showed the most severe damage. It is of particular interest that there was no involvement at the T4 level which is believed to be the site of greatest vulnerability in hypoperfusion syndromes. ${ }^{10}$ The fact that the central grey matter became selectively necrotic supports Thompson's postulate of an order of vulnerability within the spinal cord in which the central grey matter is the most vulnerable element. ${ }^{1}$ Animal experiments have supported this selective vulnerability of the central grey matter to hypoxia ${ }^{11}$ and occlusion of the aorta. ${ }^{12}$

In conclusion, it appears that severe systemic 
hypotension can produce central cord necrosis in addition to the more common mechanisms of arterial occlusion and circulatory arrest.

We are indebted to Professor H Urich for helpful advice and criticism.

\section{References}

1 Thompson GB. Dissecting aortic aneurysm with infarction of the spinal cord. Brain 1956; 79: 111-8.

2 Kepes JJ. Selective necrosis of spinal cord grey matter. A complication of dissecting aneurysm of the aorta. Acta Neuropathol 1965; 4:293-8.

3 Moersch FF, Sayre GP. Neurologic manifestations associated with dissecting aneurysm of the aorta. JAMA 1950; 144:1141-8.

4 Beattie EJ, Nolan J, Howe JS. Paralysis following surgical correction of coarctation of the aorta. Surgery 1953; 33:754-60.

5 Herrick MK, Mills PE. Infarction of spinal cord. Two cases of selective grey matter involvement secondary to asymptomatic aortic disease. Arch
Neurol 1971; 24:228-41.

6 Gilles FH, Nag D. Vulnerability of human spinal cord in transient cardiac arrest. Neurology (Minneap) 1971; 21:833-9.

7 Schneider H, Dralle J, Ebhardt G. Läsionen des Rückenmarks nach temporärem Kreislaufstillstand. Z Neurol 1973; 204:165-1978.

8 Azzarelli B, Roessmann V. Diffuse "anoxic" myelopathy. Neurology (Minneap) 1977; 27: 1049-52.

9 Madow L, Alpers BJ. Involvement of the spinal cord in occlusion of the coronary vessels. Arch Neurol 1949; 61:430-8.

10 Zülch KJ, Behrend RCH. The pathogenesis and topography of anoxia, hypoxia and ischaemia of the brain in man. In: Meyer JS, Gastaut $\mathrm{H}$ eds: Cerebral anoxia and electroencephalogram. Springfield: Charles C Thomas, 1963: p144-63.

11 Gelfan S, Tarlov IM. Differential vulnerability of spinal cord structures to anoxia. J Neurophysiol 1955; 18:170-88.

12 Krogh E. Studies on the blood supply to certain regions in the lumbar part of the spinal cord. Acta Physiol Scand 1945; 10:271-81. 